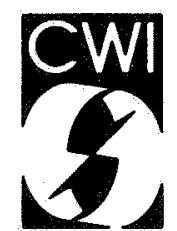

Centrum voor Wiskunde en Informatica Centre for Mathematics and Computer Science

J.L. van den Berg, O.J. Boxma

Sojourn times in feedback queues

就

Department of Operations Research and Sytem Theory

Report OS-R8710

June 
The Centre for Mathematics and Computer Science is a research institute of the Stichting Mathematisch Centrum, which was founded on February 11, 1946, as a nonprofit institution aiming at the promotion of mathematics, computer science, and their applications. It is sponsored by the Dutch Government through the Netherlands Organization for the Advancement of Pure Research (Z.W.O.).

Copyright $\odot$ Stichting Mathematisch Centrum, Amsterdam 


\title{
Sojourn Times in Feedback Queues
}

\author{
J.L. van den Berg, O.J. Boxma \\ Centre for Mathematics and Computer Science \\ P.O. Box 4079, 1009 AB Amsterdam, The Netherlands
}

\begin{abstract}
This paper considers an $M / M / 1$ queue with a very general feedback mechanism. When a customer completes his $i$-th service, he departs from the system with probability $1-p(I)$ and he cycles back with probability $p(i)$. The main result of the paper is a formula for the joint distribution of the successive sojourn times of a customer in the system. As a by-result, it is shown that the sojourn times in all individual cycles are identically, negative exponentially, distributed. Also, the correlation between the sojourn times of the $j-$ th and $k$-th cycle of a customer is calculated; furthermore, the distribution of the total sojourn time is derived.
\end{abstract}

1980 Mathematics Subject Classification: 60K25, 68M20.
Key Words \& Phrases: M/M/1 queue, feedback, sojourn times.
Note: This report will be submitted for publication elsewhere.

\section{INTRODUCTION}

This paper considers an $M / M / 1$ queue with a very general feedback mechanism. When a newly arriving customer, to be called a type-1 customer, has received his service, he departs from the system with probability $1-p(1)$ and is fed back to the end of the queue with probability $p(1)$; in the latter case he becomes a type- 2 customer. When he has received his $i$-th service, he leaves with probability $1-p(i)$ and he cycles back with probability $p(i)$, in the latter case becoming a type- $i+1$ customer. The service times of each customer at all visits are identically, negative exponentially, distributed stochastic variables. The resulting queueing model has the property that the joint queue-length distribution of type- $i$ customers, $i=1,2, \ldots$, is of product-form type. This property is exploited to analyse the sojourn-time process. In particular, we are interested in the joint distribution of the sojourn times of a customer on his successive cycles.

Feedback queues are useful for modelling many phenomena in computer-communication systems. The following example in computer timesharing was communicated to us by R.D. Nelson (IBM Research Center, Hawthorne). A newly arriving job is considered to be a class 1 job. The scheduler first allocates some CPU time to the job. During this initial CPU allocation the job, if not completed, establishes its initial working set and typically changes class to class 2 . The next time the job obtains the processor it is allocated a CPU slice for class 2 customers and, if not finished, becomes a class 3 job with its associated CPU slice. This procedure continues until the job leaves the system. This example gives rise to a single server queue with feedback. R.D. Nelson [7] considers both the case in which jobs of all classes form one queue which is processed in FCFS order, and the case of a headof-the-line priority scheme. For both cases, allowing general service time distributions, he has obtained a set of equations for the mean sojourn times during each cycle. This set of equations can be solved numerically.

Other examples of feedback systems are found in telecommunications. E.g., a telephone call may generate several tasks for processing. Such tasks can sometimes be considered as feedbacks. From a customer's viewpoint, a very important performance measure is the response time or sojourn time, defined as the time spent by a particular sequence of tasks from its arrival to its service completion [6]; and not just its mean is of interest, but also (the tail of) its distribution.

In the queueing literature, research on feedback queues has been mainly restricted to single-server queues with so-called Bernoulli feedback [3,4,9]: when a customer (task) completes his service, he departs from the system with probability $1-p$ and is fed back with probability $p$. Fontana and Diaz

Report OB-R8710

Centre for Mathematics and Computer Science

P.O. Box 4079, 1009 AB Amsterdam, The Netherlands 
Berzosa [5,6] extend some results obtained for the $M / G / 1$ model with Bernoulli feedback to a more general feedback model with priorities. In [2] we have considered sojourn time distributions in an $\mathrm{M} / \mathrm{M} / 1$ queue with deterministic feedback, viz., each customer makes exactly $N$ passes through the system. Simultaneously, Doshi and Kaufman [4] have studied sojourn time distributions in an M/G/1 queue with Bernoulli feedback, employing an iteration procedure which is very similar to the one used in [2]. The feedback mechanisms in those studies are special cases of the one in the present study: for deterministic feedback, take $p(i)=1, i \leqslant N-1, p(N)=0$; for Bernoulli feedback, take $p(i) \equiv p$.

The organization of the rest of the paper is as follows. In Section 2 the model is described in detail. Section 3 contains our main result. We derive a formula for (the transform of) the joint distribution of the successive sojourn times of a customer in the system and the number of customers of the various types present at his successive departure epochs. As a by-result, it is shown that the sojourn times in all individual cycles are identically, negative exponentially, distributed. Also, the correlation between the sojourn times of the $j$-th and $k$-th cycle of a customer is calculated; furthermore, the distribution of the total sojourn time is derived. In Section 4 two special feedback mechanisms are studied: Bernoulli feedback (Subsection 4.1) and deterministic feedback (Subsection 4.2). In these two cases, a limiting procedure leads to the sojourn time distribution in the $M / M / 1$ queue with processor sharing and the $M / D / 1$ queue with processor sharing, respectively.

\section{MODEL DESCRIPTION}

We consider a single server queueing system with infinite waiting room, see Fig. 1. Customers arrive at the system according to a Poisson process with intensity $\lambda>0$. After having received a service, a customer may either leave the system or be fed back. When a customer has completed his $i$-th service, he departs from the system with probability $1-p(i)$ and is fed back with probability $p(i)$. Fed back customers return instantaneously, joining the end of the queue. A customer who is visiting the queue for the $i$-th time will be called a type-i customer. To avoid the problems that occur in dealing with an infinite number of customer types, it is assumed that after a certain number of services the feedback probabilities of a customer remain constant. Thus $p(i)=p(N):=p, i=N, N+1, \ldots$ for some $N \geqslant 1$. The service discipline is First Come First Served (FCFS).

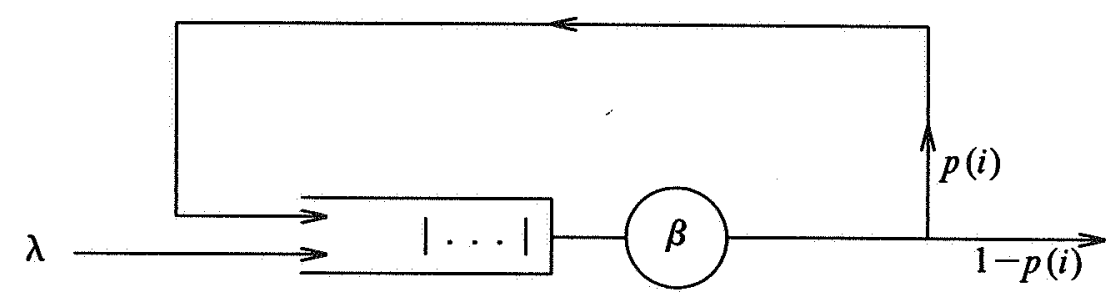

Fig. $1 \mathrm{M} / \mathrm{M} / 1$ queue with feedback.

The successive service times of a customer are independent, negative exponentially distributed, random variables, with mean $\beta$. These service times are also independent of the service times of other customers.

Introduce

$$
\begin{aligned}
& q(0):=1, \\
& q(i):=\prod_{j=0}^{i-1} p(j), \quad i=1, \ldots, N-1,
\end{aligned}
$$


with

$$
q(N):=\sum_{m=N}^{\infty} \prod_{j=0}^{m-1} p(j)=q(N-1) p(N-1) /(1-p)
$$

$$
p(0):=1 \text {. }
$$

Note that $q(i)$ is the relative arrival rate of type-i customers, $i=1, \ldots, N$. The offered load to the queue per unit of time is $\rho:=\lambda \beta \sum_{i=1}^{N} q(i)$. For stability it is required that $\rho<1$.

We are interested in the following quantities:

$-\mathrm{X}_{i}$ : number of type- $i$ customers in the system at an arbitrary epoch, $i=1, \ldots, N$;

- $\mathbf{X}_{i}^{(j)}:$ number of type-i customers in the system at the $j$-th service completion of a customer,

$$
i=1, \ldots, N, j=1,2, \ldots
$$

$-\mathrm{X}_{i}^{(0)}:$ number of type- $i$ customers in the system at the arrival of a new customer, $i=1, \ldots, N$;

- $\mathbf{S}_{j}$ : time required for the $j$-th pass through the system ( $j$-th sojourn time), $j=1,2, \ldots$.

\section{MAIN RESULtS}

In this section we present, in the form of Laplace-Stieltjes transforms and generating functions, an expression for the joint distribution of the successive sojourn times $\mathbf{S}_{j}, j=1, \ldots, k$, and the number of type-i customers, $\mathbf{X}_{l}^{(j)}, i=1, \ldots, N$, present at the $j$-th service completion of a customer who is fed back at least $k-1$ times, $k=1,2, \ldots$. First note that the system described above can be considered as a queueing network consisting of one queue with $N$ types of customers. Type- $i$ customers are fed back with probability $p(i)$ after service, and then change into type- $(i+1)$ customers, $i=1, \ldots, N-1$. Type$N$ customers are fed back with probability $p$ after service, and do not change their type. Because the service times are assumed to be exponentially distributed, the results obtained by Baskett et al. [1] can be applied to find the joint distribution of the number of type- $i$ customers in the system at an arbitrary epoch. It is found that, for $x_{1}, \ldots, x_{N}=0,1, \ldots$,

$$
\begin{aligned}
& P\left(x_{1}, \ldots, x_{N}\right):=\operatorname{Pr}\left\{\mathrm{X}_{1}=x_{1}, \ldots, \mathrm{X}_{N}=x_{N}\right\}= \\
& \left(1-\lambda \beta \sum_{i=1}^{N} q(i)\right) \prod_{i=1}^{N}(\lambda \beta q(i))^{x_{i}} \frac{\left(x_{1}+\ldots+x_{N}\right) !}{x_{1} ! \cdots x_{N} !}
\end{aligned}
$$

The PASTA property ([10]) implies the equality of the joint queue length distribution at the epoch of a new arrival and at an arbitrary epoch:

$$
\operatorname{Pr}\left\{\mathbf{X}_{1}^{(0)}=x_{1}, \ldots, \mathbf{X}_{N}^{(0)}=x_{N}\right\}=P\left(x_{1}, \ldots, x_{N}\right), \quad x_{1}, \ldots, x_{N}=0,1, \ldots
$$

We follow a customer from the moment he arrives as a type-1 customer until he completes his $k$-th service. It holds that

$$
\begin{aligned}
& E\left\{e^{-\left(\omega_{1} \mathbf{S}_{1}+\ldots+\omega_{k} \mathbf{S}_{k}\right)}\left(z_{1,0}^{\mathbf{X}_{1}^{(0)}} \cdots z_{N, 0}^{\mathbf{X}_{N}^{(0)}}\right) \cdots\left(z_{1, k}^{\mathbf{X}_{1}^{(k)}} \cdots z_{N, k}^{\mathbf{X}_{N}^{(k)}}\right)\right\}= \\
& \sum_{x_{1}=0}^{\infty} \cdots \sum_{x_{N}=0}^{\infty} P\left(x_{1}, \ldots, x_{N}\right) E\left\{e^{-\left(\omega_{1} \mathbf{S}_{1}+\ldots+\omega_{k} \mathbf{S}_{k}\right)}\left(z_{1,0}^{\mathbf{X}_{1}^{(0)}} \cdots z_{N, 0}^{\mathbf{X}_{N}^{(0)}}\right) \cdots\left(z_{1, k}^{\mathbf{X}_{1}^{(k)}} \cdots z_{N, k}^{\mathbf{X}_{N}^{(k)}}\right) \mid \mathbf{X}_{1}^{(0)}=x_{1}, \ldots, \mathbf{X}_{N}^{(0)}=x_{N}\right\}
\end{aligned}
$$


Using the property that $\mathbf{S}_{j}$ is fully determined by the number of fed back customers and the number of external arrivals during $\mathbf{S}_{j-1}$, it is shown in the Appendix that

$$
\begin{aligned}
& E\left\{e^{-\left(\omega_{1} \mathbf{S}_{1}+\ldots+\omega_{k} \mathbf{S}_{k}\right)}\left(z_{1,0}^{\mathbf{X}_{1}^{(0)}} \cdots z_{N, 0}^{\mathbf{X}_{N}^{(0)}}\right) \cdots\left(z_{1, k}^{\mathbf{X}_{1}^{(k)}} \cdots z_{N, k}^{\mathbf{X}_{N}^{(k)}}\right) \mid \mathbf{X}_{1}^{(0)}=x_{1}, \ldots, \mathbf{X}_{N}^{(0)}=x_{N}\right\}= \\
& \prod_{j=1}^{k} A_{k}^{N}(j, \omega, z) \prod_{i=1}^{N}\left(z_{i, 0} f_{k}^{N}(i, \omega, z)\right)^{x_{i}},
\end{aligned}
$$

with $\omega:=\left(\omega_{1}, \ldots, \omega_{k}\right), z:=\left(\left(z_{1,0}, \ldots, z_{N, 0}\right), \ldots,\left(z_{1, k}, \ldots, z_{N, k}\right)\right)$, and

$$
\begin{aligned}
A_{k}^{N}(1, \omega, z) & :=\left[1+\beta\left\{\omega_{k}+\lambda\left(1-z_{1, k}\right)\right\}\right]^{-1}, \\
A_{k}^{N}(2, \omega, z) & :=\left[1+\beta\left\{\omega_{k-1}+\lambda-\lambda z_{1, k-1} A_{k}^{N}(1, \omega, z)\left[p(1) z_{2, k}+1-p(1)\right]\right\}\right]^{-1}, \\
A_{k}^{N}(i, \omega, z) & :=\left[1+\beta\left\{\omega_{k-i+1}+\lambda-\lambda z_{1, k-i+1} A_{k}^{N}(i-1, \omega, z)\left[A _ { k } ^ { N } ( i - 2 , \omega , z ) \left[\cdots \left[A _ { k } ^ { N } ( 2 , \omega , z ) \left[A_{k}^{N}(1, \omega, z)\right.\right.\right.\right.\right.\right. \\
& {\left.\left.\left.\left[p(i-1) z_{i, k}+1-p(i-1)\right] p(i-2) z_{i-1, k-1}+1-p(i-2)\right] p(i-3) z_{i-2, k-2}+1-p(i-3)\right] \cdots\right] } \\
& \left.\left.\left.p(1) z_{2, k-i+2}+1-p(1)\right]\right\}\right]^{-1}, \quad i=3, \ldots, k, \\
f_{k}^{N}(i, \omega, z) & :=A_{k}^{N}(k, \omega, z)\left[A _ { k } ^ { N } ( k - 1 , \omega , z ) \left[\cdots \left[A _ { k } ^ { N } ( 2 , \omega , z ) \left[A_{k}^{N}(1, \omega, z)\right.\right.\right.\right. \\
& {\left.\left[p(k+i-1) z_{k+i, k}+1-p(k+i-1)\right] p(k+i-2) z_{k+i-1, k-1}+1-p(k+i-2)\right] } \\
& \left.\left.\left.p(k+i-3) z_{k+i-2, k-2}+1-p(k+i-3)\right] \cdots\right] p(i) z_{i+1,1}+1-p(i)\right], \quad i=1, \ldots, N .
\end{aligned}
$$

Here we have defined $z_{i, j}:=z_{N, j}, i=N+1, \ldots, N+k$.

Substituting (3.4) and (3.1) into (3.3) we obtain our main result:

\section{THEOREM}

$$
E\left\{e^{-\left(\omega_{1} \mathbf{S}_{1}+\ldots+\omega_{k} \mathbf{S}_{k}\right)}\left(z_{1,0}^{\mathbf{X}_{1}^{(0)}} \cdots z_{N, 0}^{\mathbf{X}_{N}^{(0)}}\right) \cdots\left(z_{1, k}^{\mathbf{X}_{1}^{(k)}} \cdots z_{N, k}^{\mathbf{X}_{N}^{(k)}}\right)\right\}=\frac{\left(1-\lambda \beta \sum_{i=1}^{N} q(i)\right) \prod_{j=1}^{k} A_{k}^{N}(j, \omega, z)}{1-\lambda \beta \sum_{i=1}^{N} q(i) z_{i, 0} f_{k}^{N}(i, \omega, z)}
$$

$$
\operatorname{Re} \omega_{j} \geqslant 0, \quad\left|z_{i, j}\right| \leqslant 1, i=1, \ldots, N, j=1, \ldots, k
$$

\section{COROLlaRY 1}

The Laplace-Stieltjes transform of the joint distribution of the first $k$ successive sojourn times of a customer, who is fed back at least $k-1$ times, is given by

$$
E\left\{e^{-\left(\omega_{1} \mathbf{S}_{1}+\ldots+\omega_{k} \mathbf{S}_{k}\right)}\right\}=\frac{\left(1-\lambda \beta \sum_{i=1}^{N} q(i)\right) \prod_{i=1}^{k} A_{k}^{N}(j, \omega)}{1-\lambda \beta \sum_{i=1}^{N} q(i) f_{k}^{N}(i, \omega)}
$$


with,

$A_{k}^{N}(1, \omega):=\left[1+\beta \omega_{k}\right]^{-1}$

$A_{k}^{N}(2, \omega):=\left[1+\beta\left\{\omega_{k-1}+\lambda-\lambda A_{k}^{N}(1, \omega)\right\}\right]^{-1}$,

$A_{k}^{N}(i, \omega):=\left[1+\beta\left\{\omega_{k-i+1}+\lambda-\lambda A_{k}^{N}(i-1, \omega)\left[A_{k}^{N}(i-2, \omega)\left[\cdots\left[A_{k}^{N}(2, \omega)\left[A_{k}^{N}(1, \omega)\right.\right.\right.\right.\right.\right.$

$p(i-2)+1-p(i-2)] p(i-3)+1-p(i-3)] \cdots] p(1)+1-p(1)]\}]^{-1}, \quad i=3, \ldots, k$,

$f_{k}^{N}(i, \omega):=A_{k}^{N}(k, \omega)\left[A_{k}^{N}(k-1, \omega)\left[\cdots\left[A_{k}^{N}(2, \omega)\left[A_{k}^{N}(1, \omega) p(k+i-2)+1-p(k+i-2)\right]\right.\right.\right.$

$p(k+i-3)+1-p(k+i-3)] \cdots p(i)+1-p(i)], \quad i=1, \ldots, N$.

ProOF: Straightforward substitution.

COROLlaRY 2

$E\left\{z_{1}^{\mathbf{X}_{1}^{(n}} \cdots z_{N}^{\mathbf{X}_{N}^{(n)}}\right\}=E\left\{z_{1}^{\mathbf{X}_{1}} \cdots z_{N}^{\mathbf{X}_{N}}\right\}=\frac{1-\lambda \beta \sum_{i=1}^{N} q(i)}{1-\lambda \beta \sum_{i=1}^{N} q(i) z_{i}},\left|z_{i}\right| \leqslant 1, i=1, \ldots, N, j=0,1, \ldots, k$.

Proof: It follows from (3.1) and (3.2) that (3.11) holds for $j=0$. If (3.11) also holds for $j=1$, then it clearly holds for all $j=0,1, \ldots$. The validity of (3.11) for $j=1$ follows by a simple calculation.

REMARKS

(i) The Laplace-Stieltjes transform of the joint sojourn time distribution can be presented in a form which is more suitable for obtaining sojourn time moments. For this purpose we first rewrite (3.9) and (3.10):

$A_{k}^{N}(i, \omega):=\left[1+\beta \omega_{k-i+1}+\lambda \beta\left\{1-\tilde{q}(i-1) \prod_{j=1}^{i-1} A_{k}^{N}(j, \omega)-\sum_{l=2}^{i-1} \tilde{q}(i-l)(1-p(i-l)) \prod_{j=l}^{i-1} A_{k}^{N}(j, \omega)\right\}\right]^{-1}, \quad i=1, \ldots, k$,

$f_{k}^{N}(i, \omega):=\frac{1}{\tilde{q}(i)}\left[\tilde{q}(k+i-1) \prod_{j=1}^{k} A_{k}^{N}(j, \omega)+\sum_{l=2}^{k} \tilde{q}(k+i-l)(1-p(k+i-l)) \prod_{j=l}^{k} A_{k}^{N}(j, \omega)\right], \quad i=1, \ldots, N$,

with

$$
\begin{aligned}
& \tilde{q}(0):=1, \\
& \tilde{q}(i):=\prod_{j=0}^{i-1} p(j), \quad i=1,2, \ldots .
\end{aligned}
$$

Introducing

$$
M(i, \omega):=\prod_{j=1}^{i} \frac{1}{A_{k}^{N}(j, \omega)}, \quad i=1, \ldots, k
$$




$$
M(0, \omega):=1,
$$

and

$$
H(k, i, \omega):=M(k, \omega) f_{k}^{N}(i, \omega), \quad i=1, \ldots, N
$$

it is easily seen that (3.8) can be written as

$$
E\left\{e^{-\left(\omega_{1} \mathbf{S}_{1}+\ldots+\omega_{k} \mathbf{S}_{k}\right)}\right\}=\frac{1-\lambda \beta \sum_{i=1}^{N} q(i)}{M(k, \omega)-\lambda \beta \sum_{i=1}^{N} q(i) H(k, i, \omega)}
$$

with

$$
\begin{aligned}
& M(i, \omega)=\left(1+\beta \omega_{k-i+1}\right) M(i-1, \omega)+\lambda \beta[ M(i-1, \omega)-\tilde{q}(i-1)- \\
&\left.\sum_{j=2}^{i-1} \tilde{q}(i-j)(1-p(i-j)) M(j-1, \omega)\right], \quad i=1, \ldots, k, \\
& H(k, i, \omega)=\frac{1}{\tilde{q}(i)}\left[\tilde{q}(k+i-1)+\sum_{j=2}^{k} \tilde{q}(k+i-j)(1-p(k+i-j)) M(j-1, \omega)\right], \quad i=1, \ldots, N .
\end{aligned}
$$

(ii) The fact that the joint queue length distribution at the arrival of a customer and after each of his passes is the same (cf. Corollary 2), implies that the sojourn times $\mathbf{S}_{j}, j=1, \ldots, k$ have the same marginal distribution. $S_{1}$ can easily be obtained from (3.13), (3.14) and (3.15) by taking $k=1$ (or taking $\left.\omega_{j}=0, j=2, \ldots, k\right)$. It is found, with offered load $\rho=\lambda \beta \sum_{i=1}^{N} q(i)$, that the sojourn times are negative exponentially distributed with mean $\beta /(1-\rho)$ :

$$
E\left\{e^{-\omega_{j} S_{j}}\right\}=\frac{1-\rho}{1-\rho+\beta \omega_{j}}, j=1, \ldots, k
$$

This coincides with the sojourn time transform in an ordinary $M / M / 1$ queue with mean service time $\beta$ and arrival rate $\lambda \sum_{i=1}^{N} q(i)$.

(iii) In order to investigate the dependence between the $i$-th and $j$-th sojourn times we have computed the Laplace-Stieltjes transform of the joint distribution of $\mathbf{S}_{i}$ and $\mathbf{S}_{j}, 1 \leqslant i<j \leqslant k$. It is found from (3.13), (3.14) and (3.15) that

$$
E\left\{e^{-\left(\omega_{i} \mathbf{S}_{1}+\omega_{j} \mathbf{S}_{j}\right)}\right\}=\frac{1-\rho}{1-\rho+\beta \omega_{i}+\beta \omega_{j}+\beta^{2} \omega_{i} \omega_{j} C_{j-i}}, \quad 1 \leqslant i<j \leqslant k,
$$

where $C_{j-i}$ is determined by

$$
\begin{aligned}
& C_{1}=1, \\
& C_{n}=(1+\lambda \beta) C_{n-1}-\lambda \beta \sum_{l=2}^{n-1} \tilde{q}(n-l)(1-p(n-l)) C_{l-1}, \quad n=2, \ldots, k-1,
\end{aligned}
$$

Note that $E\left\{e^{-\left(\omega_{i} \mathbf{S}_{i}+\omega_{j} \mathbf{S}_{j}\right)}\right\}$ only depends on $i$ and $j$ through the difference $j-i$. This property might also have been derived from Corollary 2 .

From (3.17) the correlation coefficient, $\operatorname{corr}\left(\mathbf{S}_{i}, \mathbf{S}_{j}\right)$, can easily be obtained:

$$
\operatorname{corr}\left(\mathbf{S}_{i}, \mathbf{S}_{j}\right)=1-C_{j-i}(1-\rho), \quad 1 \leqslant i<j \leqslant k \text {. }
$$


It follows from (3.18) and (3.19) that $\operatorname{corr}\left(\mathbf{S}_{i}, \mathbf{S}_{j}\right)$ as a function of $i$ and $j$ only depends on $j-i$, and that it decreases if $j-i$ grows. For $j-i=1, \operatorname{corr}\left(\mathbf{S}_{i}, \mathbf{S}_{j}\right)=\rho$.

(iv) The Laplace-Stieltjes transform of the distribution of a customer's total time spent in the system until the end of his $k$-th pass $\mathbf{S}^{(k)}:=\mathbf{S}_{1}+\ldots+\mathbf{S}_{k}$, can be obtained from (3.13) by substituting $\omega_{j}=\omega_{0}, j=1, \ldots, k$. To derive an expression for the variance of this sojourn time, $\operatorname{var}\left(\mathbf{S}^{(k)}\right)$, it is convenient to use the formula

$$
\operatorname{var}\left(\mathbf{S}^{(k)}\right)=k \operatorname{var}\left(\mathbf{S}_{1}\right)+2 \sum_{i=1}^{k} \sum_{j=i+1}^{k} \operatorname{cov}\left(\mathbf{S}_{i}, \mathbf{S}_{j}\right)
$$

The covariance of $\mathbf{S}_{i}$ and $\mathbf{S}_{j}, \operatorname{cov}\left(\mathbf{S}_{i}, \mathbf{S}_{j}\right)$, and $\operatorname{var}\left(\mathbf{S}_{1}\right)$ can easily be obtained from the results given in (ii) and (iii). It is found that

$$
\operatorname{var}\left(\mathbf{S}^{(k)}\right)=\left(\frac{\beta}{1-\rho}\right)^{2}\left[k^{2}-2(1-\rho) \sum_{j=1}^{k-1} j C_{k-j}\right],
$$

with $C_{1}, \ldots, C_{k-1}$ given by (3.18).

The Laplace-Stieltjes transform of the distribution of the total sojourn time $\mathbf{S}$ of an (arbitrary) customer is now given by

$$
E\left\{e^{-\omega_{0} \mathbf{S}}\right\}=\sum_{k=1}^{\infty} \tilde{q}(k)(1-p(k)) E\left\{e^{-\omega_{0} \mathbf{S}^{(k)}}\right\}
$$

(v) Let the mean service time $\beta \downarrow 0$, and choose the feedback probabilities such that the total required mean service time, $\tilde{\beta}$, remains a positive constant. It is intuitively clear that this limiting procedure reduces the feedback queue to an $M / G / 1$ queue with processor sharing. Different choices of the feedback probabilities lead to different total service time distributions with mean $\tilde{\beta}$. This opens the possibility for a new derivation of sojourn time results for the $M / G / 1$ queue with processor sharing (cf. Ott [8]). We shall not pursue this here; in Section 4 the $M / D / 1$ processor sharing queue will thus be analyzed.

\section{EXAMPLE}

In this example we examine the case $k=2$ for which explicit closed form results are easily obtained. From (3.17) and (3.18) it follows that for this case

$$
E\left\{e^{-\left(\omega_{1} S_{1}+\omega_{2} S_{2}\right)}\right\}=\frac{1-\rho}{1-\rho+\beta \omega_{1}+\beta \omega_{2}+\beta^{2} \omega_{1} \omega_{2}} .
$$

Note that the feedback probabilities $p(i), i=1, \ldots, N$, enter into the joint distribution of $S_{1}$ and $S_{2}$ only via the offered load $\rho$. Thus, as long as $\rho$ remains constant, the joint distribution of $S_{1}$ and $S_{2}$ is independent of the individual values of $p(i), i=1, \ldots, N$. Doshi and Kaufman [4] derived (3.22) for the (special) case of Bernoulli feedback $(p(i) \equiv p)$.

From (3.19) it follows that

$$
\operatorname{corr}\left(\mathbf{S}_{1}, \mathbf{S}_{2}\right)=\rho .
$$

Let $F_{2}(\cdot)$ denote the distribution function of the sojourn time until the end of the second pass: 


$$
F_{2}(t):=\operatorname{Pr}\left\{\mathrm{S}_{1}+\mathrm{S}_{2}<t\right\}, \quad t \geqslant 0 .
$$

From (3.22) we find

$$
E\left\{e^{-\omega_{0}\left(\mathbf{S}_{1}+\mathbf{S}_{2}\right)}\right\}=\frac{1+\sqrt{\rho}}{2 \sqrt{\rho}} \frac{1-\sqrt{\rho}}{1-\sqrt{\rho}+\beta \omega_{0}}-\frac{1-\sqrt{\rho}}{2 \sqrt{\rho}} \frac{1+\sqrt{\rho}}{1+\sqrt{\rho}+\beta \omega_{0}} .
$$

Hence

$$
F_{2}(t)=\frac{1+\sqrt{\rho}}{2 \sqrt{\rho}}\left(1-e^{-t(1-\sqrt{\rho}) / \beta}\right)-\frac{1-\sqrt{\rho}}{2 \sqrt{\rho}}\left(1-e^{-t(1+\sqrt{\rho}) / \beta}\right), \quad t \geqslant 0 .
$$

In [4] Doshi and Kaufman compare $F_{2}(\cdot)$ with the distribution of $\mathbf{S}_{1}+\mathbf{S}_{2}$ that results when one assumes that $\mathbf{S}_{1}$ and $\mathbf{S}_{2}$ are independent. Due to the positive correlation between $\mathbf{S}_{1}$ and $\mathbf{S}_{2}$ (cf. (3.23)), it is found that $F_{2}(\cdot)$ has a longer "tail" than this approximate distribution.

Finally, the variance of $S_{1}+S_{2}$ is obtained from (3.20):

$$
\operatorname{var}\left(\mathbf{S}_{1}+\mathrm{S}_{2}\right)=2(1+\rho)\left(\frac{\beta}{1-\rho}\right)^{2}
$$

\section{SPECial cases}

In this section we study two feedback systems which are special cases of the general model described in Section 2, viz., Bernoulli feedback (Subsection 4.1) and deterministic feedback (Subsection 4.2). It will appear that these models yield simple, explicit expressions for most of the quantities analyzed in Section 3. At the end of Subsection 4.2 we show that the sojourn time distribution in the $M / D / 1$ queue with processor sharing can easily be obtained from the sojourn time distribution in the deterministic feedback model.

The Laplace-Stieltjes transform of the joint sojourn time distribution in the Bernoulli feedback model has also been derived by Doshi and Kaufman [4]. The results for the deterministic feedback model were already obtained in a previous paper [2]. In fact, those results have led to the study of the general model.

\subsection{Bernoulli feedback}

The Bernoulli feedback model is obtained from the general model by taking $N=1$ : when a customer completes his service he departs from the system with probability $1-p$ and is fed back with probability $p$.

Obviously

$$
\rho=\frac{\lambda \beta}{1-p}
$$

The Laplace-Stieltjes transform of the joint distribution of the successive sojourn times $\mathbf{S}_{1}, \ldots, \mathbf{S}_{k}$ can be obtained from (3.8)-(3.10) by substituting $N=1, p(i) \equiv p$. The resulting expression has also been derived by Doshi and Kaufman [4].

To obtain explicit expressions for $E\left\{e^{-\left(\omega_{i} \mathbf{S}_{i}+\omega_{j} \mathbf{S}_{j}\right)}\right\}, \operatorname{corr}\left(\mathbf{S}_{i}, \mathbf{S}_{j}\right)$, and $\operatorname{var}\left(\mathbf{S}^{(k)}\right)$, (see (3.17), (3.19) and (3.20)) we have to derive $C_{n}, n=1, \ldots, k-1$, from the set of difference equations (3.18). After the substitution $\tilde{q}(j)=p^{j-1}, j=1,2, \ldots,(3.18)$ reduces to

$$
\begin{aligned}
& C_{1}=1, \\
& C_{n}=(1+\lambda \beta) C_{n-1}-\lambda \beta \sum_{l=2}^{n-1}\left(p^{n-l-1}-p^{n-l}\right) C_{l-1}, \quad n=2, \ldots, k-1 .
\end{aligned}
$$


From (4.1) it follows that

$$
\begin{aligned}
& C_{1}=1 \\
& C_{2}=1+\lambda \beta, \\
& C_{n}-p C_{n-1}=(1+\lambda \beta) C_{n-1}-p(1+\lambda \beta) C_{n-2}-\lambda \beta(1-p) C_{n-2}, \quad n=3, \ldots, k-1 .
\end{aligned}
$$

Hence

$$
\begin{aligned}
& C_{1}=1 \\
& C_{2}=1+\lambda \beta \\
& C_{n}=(1+\lambda \beta+p) C_{n-1}-(\lambda \beta+p) C_{n-2}, \quad n=3, \ldots, k-1 .
\end{aligned}
$$

The general solution of (4.2) is given by

$$
C_{n}=U_{1} y_{1}^{n}+U_{2} y_{2}^{n}
$$

where $y_{1}=1$ and $y_{2}=\lambda \beta+p$ are the roots of

$$
y^{2}-(1+\lambda \beta+p) y+(\lambda \beta+p)=0,
$$

and $U_{1}$ and $U_{2}$ are determined by

$$
\begin{aligned}
& U_{1} y_{1}+U_{2} y_{2}=1, \\
& U_{1} y_{1}^{2}+U_{2} y_{2}^{2}=1+\lambda \beta .
\end{aligned}
$$

After some calculations it is found that

$$
C_{n}=\frac{1-p-\lambda \beta(\lambda \beta+p)^{n-1}}{1-p-\lambda \beta}, \quad n=1, \ldots, k-1
$$

Substitution of (4.3) in (3.17), (3.19) and (3.20) yields

$$
\begin{aligned}
& E\left\{e^{-\left(\omega_{i} \mathbf{S}_{i}+\omega_{,} \mathbf{S}_{j}\right)}\right\}=\frac{1-\frac{\lambda \beta}{1-p}}{1-\frac{\lambda \beta}{1-p}+\beta \omega_{i}+\beta \omega_{j}+\beta^{2} \omega_{i} \omega_{j} \frac{1-p-\lambda \beta(\lambda \beta+p)^{j-i-1}}{1-p-\lambda \beta}}, \quad 1 \leqslant i<j \leqslant k, \\
& \operatorname{corr}\left(\mathbf{S}_{i}, \mathbf{S}_{j}\right)=\frac{\lambda \beta}{1-p}(\lambda \beta+p)^{j-i-1}, \quad 1 \leqslant i<j \leqslant k \\
& \operatorname{var}\left(\mathbf{S}^{(k)}\right)=\left[\frac{\beta}{1-\frac{\lambda \beta}{1-p}}\right]^{2}\left[k+2 \frac{\lambda \beta}{(1-p)^{2}}\left\{\frac{k}{1-\frac{\lambda \beta}{1-p}}-\frac{1}{1-p} \frac{1}{\left(1-\frac{\lambda \beta}{1-p}\right)^{2}}\left(1-(p+\lambda \beta)^{k}\right)\right\}\right.
\end{aligned}
$$


It follows from (4.5) that $\lim _{j-i \uparrow \infty} \operatorname{corr}\left(\mathbf{S}_{i}, \mathbf{S}_{j}\right)=0$ (cf. Section 3, Remarks (iii)). It is also seen that $\operatorname{corr}\left(\mathbf{S}_{i}, \mathbf{S}_{j}\right)$ is an increasing function of $\lambda \beta$ for fixed $i$ and $j$. These intuitively appealing properties are illustrated in Fig. 2.

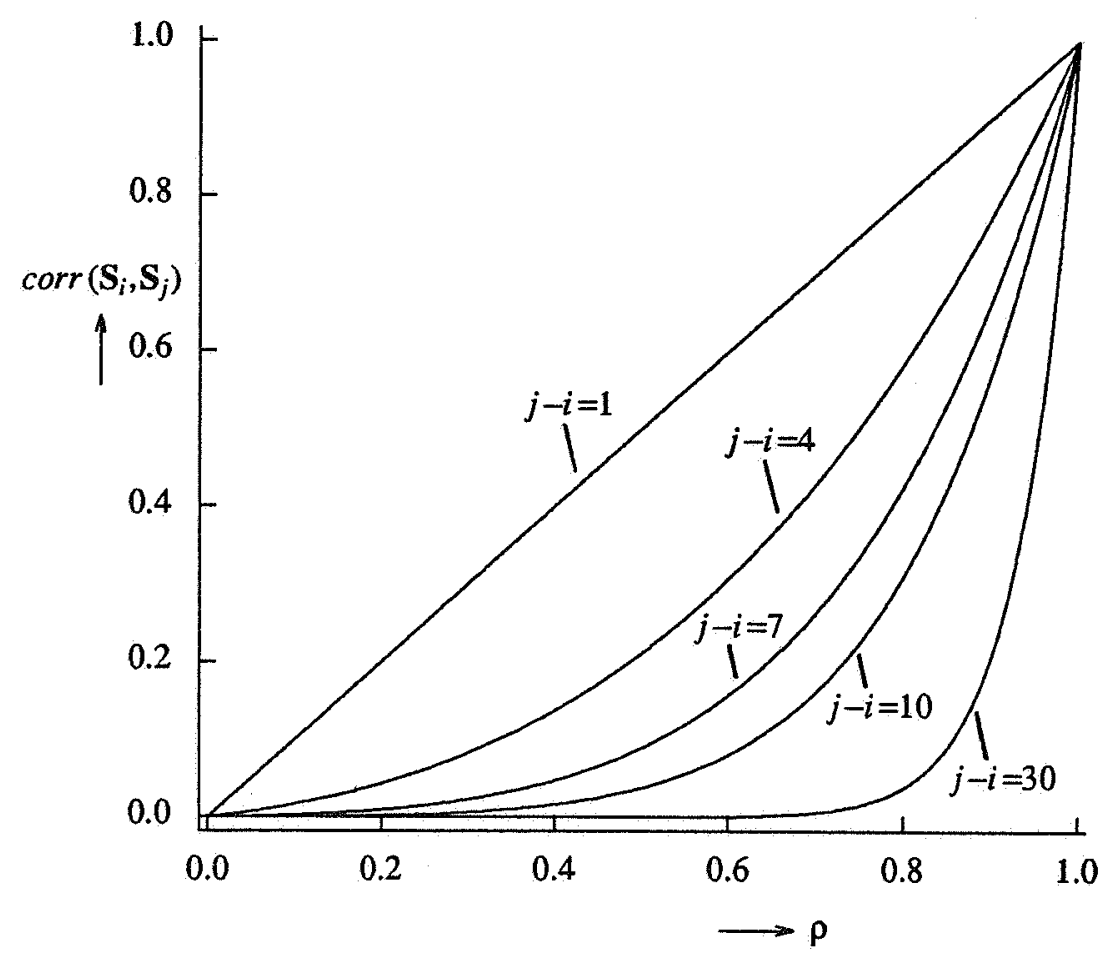

Fig. $2 \operatorname{corr}\left(\mathbf{S}_{i}, \mathbf{S}_{j}\right)$ as a function of offered load $\rho=\frac{\lambda \beta}{1-p}$, with $p=0.5$.

The Laplace-Stieltjes transform of $\mathbf{S}^{(k)}$ can be obtained from (3.13)-(3.15) by substituting $\omega_{j}=\omega_{0}, j=1, \ldots, k$. The resulting set of difference equations (3.14) can be solved in the same way as (4.2). After extensive but straightforward calculations it is found that

$$
E\left\{e^{-\omega_{0} \mathbf{S}^{(k)}}\right\}=\frac{1-p-\lambda \beta}{Q_{1}\left(1-x_{2}\right) x_{1}^{k}+Q_{2}\left(1-x_{1}\right) x_{2}^{k}}, \quad \operatorname{Re} \omega_{0} \geqslant 0, \quad k=1,2, \ldots,
$$

where,

$$
\begin{aligned}
& x_{1}=\frac{1+\beta \omega_{0}+\lambda \beta+p+\sqrt{\left(1+\beta \omega_{0}+\lambda \beta+p\right)^{2}-4\left(p+p \beta \omega_{0}+\lambda \beta\right)}}{2}, \\
& x_{2}=\frac{1+\beta \omega_{0}+\lambda \beta+p-\sqrt{\left(1+\beta \omega_{0}+\lambda \beta+p\right)^{2}-4\left(p+p \beta \omega_{0}+\lambda \beta\right)}}{2} \\
& Q_{1}=\frac{x_{2}-\left(1+\beta \omega_{0}\right)}{x_{2}-x_{1}} \\
& Q_{2}=\frac{x_{1}-\left(1+\beta \omega_{0}\right)}{x_{1}-x_{2}}
\end{aligned}
$$


Letting $p \uparrow 1$ and $\lambda \beta \downarrow 0$ such that $\lambda \beta /(1-p)$ remains constant, reduces the feedback model to the $\mathrm{M} / \mathrm{M} / 1$ queue with processor sharing. Application of this limiting procedure to the total sojourn time distribution, which readily follows from (4.7), yields the sojourn time distribution in the $M / M / 1$ queue with processor sharing (Ott [8]). The calculations, which are quite involved, are omitted here.

\subsection{Deterministic feedback}

Taking $p(i)=1, i=1, \ldots, N-1, p(N)=p=0$, we obtain the deterministic feedback model in which each customer is fed back exactly $N-1$ times. After the $N$-th service the customer leaves the system. Obviously

$$
\rho=N \lambda \beta .
$$

Noting that

$$
\begin{aligned}
q(j)=\tilde{q}(j) & =1, & & 0 \leqslant j \leqslant N, \\
& =0, & & j>N,
\end{aligned}
$$

it is easily seen from (3.13)-(3.15) that

$$
E\left\{e^{-\left(\omega_{1} \mathbf{S}_{1}+\ldots+\omega_{N} \mathbf{S}_{N}\right)}\right\}=\frac{1-N \lambda \beta}{M(N, \omega)-\lambda \beta \sum_{i=0}^{N-1} M(i, \omega)},
$$

with

$$
\begin{aligned}
& M(0, \omega)=1 \\
& M(i, \omega)=\left(1+\lambda \beta+\beta \omega_{N-i+1}\right) M(i-1, \omega)-\lambda \beta, \quad i=1, \ldots, N
\end{aligned}
$$

At the end of this section we shall use (4.8) and (4.9) to obtain the Laplace-Stieltjes transform of the total sojourn time distribution.

As in Section 4.1 we solve the set of difference equations (3.18) to obtain explicit expressions for $E\left\{e^{-\left(\omega_{i} \mathbf{S}_{i}+\omega_{j} \mathbf{S}_{j}\right)}\right\}$, $\operatorname{corr}\left(\mathbf{S}_{i}, \mathbf{S}_{j}\right)$, and $E\left\{e^{-\omega_{0} \mathbf{S}^{m}}\right\}$ from the general formulas (3.17), (3.19) and (3.20). Substituting in (3.18) $p(i)=\tilde{q}(i)=1, i=1, \ldots, N-1$, we get

$$
\begin{aligned}
& C_{1}=1, \\
& C_{n}=(1+\lambda \beta) C_{n-1}, \quad n=2, \ldots, N-1 .
\end{aligned}
$$

Hence

$$
C_{n}=(1+\lambda \beta)^{n-1}, \quad n=1, \ldots, N-1
$$

Now it follows from (3.17), (3.19) and (3.20) that

$$
\begin{aligned}
& E\left\{e^{-\left(\omega_{j} \mathbf{S}_{i}+\omega_{j} \mathbf{S}_{j}\right)}\right\}=\frac{1-N \lambda \beta}{1-N \lambda \beta+\beta \omega_{i}+\beta \omega_{j}+\beta^{2} \omega_{i} \omega_{j}(1+\lambda \beta)^{j-i-1}}, \quad 1 \leqslant i<j \leqslant N, \\
& \operatorname{corr}\left(\mathbf{S}_{i}, \mathbf{S}_{j}\right)=1-(1-N \lambda \beta)(1+\lambda \beta)^{j-i-1}, \quad 1 \leqslant i<j \leqslant N,
\end{aligned}
$$


$\operatorname{var}\left(\mathbf{S}^{(N)}\right)=\operatorname{var}(\mathbf{S})=\left[\frac{\beta}{1-N \lambda \beta}\right]^{2}\left[\frac{2}{(\lambda \beta)^{2}}-N^{2}-2(1-N \lambda \beta) \frac{(1+\lambda \beta)^{N}}{(\lambda \beta)^{2}}\right]$.

The Laplace-Stieltjes transform of the distribution of the total sojourn time $\mathbf{S}$ is obtained from (4.8) and (4.9) by substituting $\omega_{j}=\omega_{0}, j=1, \ldots, N$. This substitution yields (cf.(4.9))

$$
\begin{aligned}
& M(0, \omega)=1, \\
& M(i, \omega)=\left(1+\lambda \beta+\beta \omega_{0}\right) M(i-1, \omega)-\lambda \beta, \quad i=1, \ldots, N .
\end{aligned}
$$

Hence

$M(i, \omega)=\left(1+\lambda \beta+\beta \omega_{0}\right)^{i}-\lambda \beta \sum_{j=0}^{i-1}\left(1+\lambda \beta+\beta \omega_{0}\right)^{j}=\frac{\beta \omega_{0}}{\lambda \beta+\beta \omega_{0}}\left(1+\lambda \beta+\beta \omega_{0}\right)^{i}+\frac{\lambda \beta}{\lambda \beta+\beta \omega_{0}}, \quad i=1, \ldots, N$

Using this result it follows from (4.8) that

$$
E\left\{e^{-\omega_{0} \mathbf{S}}\right\}=\frac{(1-N \lambda \beta)\left(\lambda+\omega_{0}\right)^{2}}{\omega_{0}^{2}\left(1+\lambda \beta+\beta \omega_{0}\right)^{N}+\lambda\left(\lambda+\omega_{0}\right)(1-N \lambda \beta)+\lambda \omega_{0}}, \quad \operatorname{Re} \omega_{0} \geqslant 0 .
$$

Finally, we shall show that (4.14) can be used to obtain the sojourn time distribution in the M/D/1 queue with processor sharing (cf. Section 3 , Remarks (v)).

Let $N \uparrow \infty$ and $\beta \downarrow 0$ in such a way that $\tilde{\beta}:=N \beta$ remains constant. Then the distribution of the total service time received by each customer approaches the deterministic distribution fixed at $\tilde{\beta}$ :

$$
\lim _{\substack{N \uparrow \infty \\ \beta \downarrow 0}}\left(\frac{1}{1+\beta \omega_{0}}\right)^{N}=\lim _{N \uparrow \infty}\left(\frac{1}{1+\frac{\tilde{\beta}}{N} \omega_{0}}\right)^{N}=e^{-\tilde{\beta} \omega_{0}}, \quad \operatorname{Re} \omega_{0} \geqslant 0 .
$$

This limiting procedure apparently reduces the deterministic feedback model to the $M / D / 1$ queueing model with processor sharing. Indeed, in the limit (4.14) equals the Laplace-Stieltjes transform of the sojourn time distribution in the $M / D / 1$ system with processor sharing:

$$
\lim _{\substack{N \uparrow \infty \\ \beta \downarrow 0}} E\left\{e^{-\omega_{0} \mathbf{S}}\right\}=\frac{\left(\lambda+\omega_{0}\right)^{2}(1-\lambda \tilde{\beta}) e^{-\tilde{\beta}\left(\lambda+\omega_{0}\right)}}{\omega_{0}^{2}+\lambda\left(\lambda+2 \omega_{0}-\lambda \tilde{\beta}\left(\lambda+\omega_{0}\right)\right) e^{-\tilde{\beta}\left(\lambda+\omega_{0}\right)}}, \quad \text { Re } \omega_{0} \geqslant 0,
$$

a result previously obtained by Ott [8].

\section{REFERENCES}

1 Baskett, F., Chandy, K.M., Muntz, R.R., Palacios, F.G. (1975). Open, closed, and mixed networks of queues with different classes of customers. J. ACM. 22, 248-260.

2 VAN DeN BerG, J.L., BoXma, O.J., GroennendiJk, W.P. (1987). Sojourn times in the M/G/1 queue with deterministic feedback, Report OS-R8708, Centre for Mathematics and Computer Science, Amsterdam.

3 DisNeY, R.L., Konig, D., SCHMIDT, V. (1984). Stationary queue-length and waiting-time distributions in single-server feedback queues. Adv. Appl. Prob. 16, 437-446.

4 Doshi, B.T., Kaufman, J.S. (1987). Sojourn Time in an M/G/1 Queue with Bernoulli Feedback. Report AT\&T Bell Laboratories, Holmdel, N.J. 
5 Fontana, B., Diaz Berzosa, C. (1984). Stationary queue-length distributions in an M/G/1 queue with two non-preemptive priorities and general feedback. In: Performance of ComputerCommunication Systems, eds. H. Rudin and W. Bux. North-Holland Publ. Cy., Amsterdam, 333-347.

6 Fontana, B., Diaz Berzosa, C. (1985). M/G/1 queue with N-priorities and feedback: joint queue-length distributions and response time distribution for any particular sequence. In: Teletraffic Issues in an Advanced Information Society, ITC-11, ed. M. Akiyama. North-Holland Publ. Cy., Amsterdam, 452-458.

7 Nelson, R.D. (1987). Private communication.

8 OTT, T.J. (1984). The sojourn-time distribution in the M/G/1 queue with processor sharing. J. Appl. Prob. 21, 360-378.

9 TAKACs, L. (1963). A single-server queue with feedback. Bell System Tech. J. 42, 505-519.

10 WolfF, R.W. (1982). Poisson arrivals see time averages. Oper. Res. 30, 223-231. 


\section{APPENDIX}

In this appendix we prove Formula (3.4). For ease of notation we introduce the service time distribution function $B(t):=1-e^{-t / \beta}$

The derivation of (3.4) is based on the fact that $\left(\mathbf{X}_{1}^{(i+1)}, \ldots, \mathbf{X}_{N}^{(i+1)}\right)$, which determines the distribution of $\mathbf{S}_{i+2}$, is conditionally independent of $\left\{\left(\mathbf{X}_{1}^{(0)}, \ldots, \mathbf{X}_{N}^{(0)}\right), \ldots,\left(\mathbf{X}_{1}^{(i-1)}, \ldots, \mathbf{X}_{N}^{(i-1)}\right) ; \mathbf{S}_{1}, \ldots, \mathbf{S}_{i}\right\}$ given $\left\{\left(\mathbf{X}_{1}^{(i)}, \ldots, \mathbf{X}_{N}^{(i)}\right) ; \mathbf{S}_{i+1}\right\}, i=1, \ldots, k-1$. Using this property it is easily seen that:

$$
\begin{aligned}
& E\left\{e^{-\left(\omega_{1} \mathbf{S}_{1}+\ldots+\omega_{k} \mathbf{S}_{k}\right)}\left(z_{1,0}^{\mathbf{X}_{1}^{(0)}} \cdots z_{N, 0}^{\mathbf{X}_{N}^{(0)}}\right) \cdots\left(z_{1, k}^{\mathbf{X}_{1}^{(k)}} \cdots z_{N, k}^{\mathbf{X}_{N}^{(k)}}\right) \mid \mathbf{X}^{(0)}=x_{1}, \ldots, \mathbf{X}_{N}^{(0)}=x_{N}\right\}= \\
& z_{1,0}^{x_{1}} \cdots z_{N, 0}^{x_{N}} \int_{t_{1}=0}^{\infty} e^{-\omega_{1} t_{1}} \int_{t_{2}=0}^{\infty} e^{-\omega_{2} t_{2}} \cdots \int_{t_{k-1}=0}^{\infty} e^{-\omega_{k-1} t_{k-1}} \int_{t_{k}=0}^{\infty} e^{-\omega_{k} t_{k}} \\
& \sum_{n_{1}^{(1)}=0}^{\infty} e^{-\lambda t_{1}} \frac{\left(\lambda t_{1}\right)^{n_{1}^{(1)}}}{n_{1}^{(1)} !} z_{1,1}^{n_{1}^{(1)}}\left[\prod_{j=1}^{N} \sum_{n_{j+1}^{(1)}=0}^{x_{j}}\left[\begin{array}{c}
x_{j} \\
n_{j}^{(1)}+1
\end{array}\right] p(j)^{n_{j+1}^{(1)}}(1-p(j))^{x_{j}-n_{j+1}^{(1)}} z_{j+1,1}^{n_{j+1}^{(i)}}\right]
\end{aligned}
$$

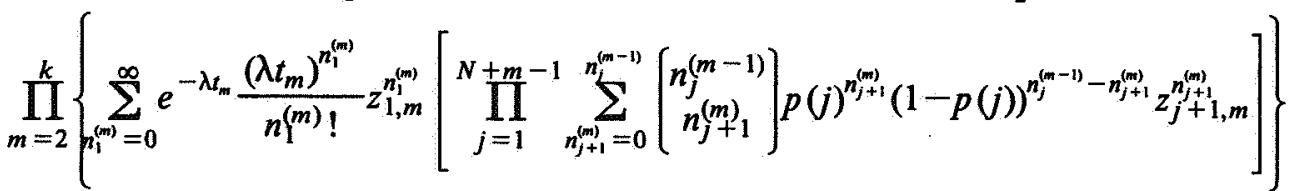

$$
\begin{aligned}
& d B\left(t_{k}\right)^{\left(1+n_{1}^{(k-1)}+\ldots+n_{k+k-1}^{(k-1)}\right)^{*}} d B\left(t_{k-1}\right)^{\left(1+n_{1}^{(k-2)}+\ldots+n_{N+k-2}^{(k-2)}\right)^{*}} \cdots d B\left(t_{2}\right)^{\left(1+n_{1}^{(1)}+\ldots+n_{N+1}^{(1)}\right)^{*}} d B\left(t_{1}\right)^{\left(1+x_{1}+\ldots+x_{k}\right)^{*}} .
\end{aligned}
$$

Note that by definition $z_{i, j}:=z_{N, j}, \quad i=N+1, \ldots, N+k, j=1, \ldots, k$.

We first evaluate the integral with respect to $t_{k}$, obtaining

$$
\begin{aligned}
& E\left\{e^{-\left(\omega_{1} \mathbf{S}_{1}+\ldots+\omega_{k} \mathbf{S}_{k}\right)}\left(z_{1,0}^{\mathbf{X}_{1}^{(0)}} \cdots z_{N, 0}^{\mathbf{X}_{N}^{(0)}}\right) \cdots\left(z_{1, k}^{\mathbf{X}_{1, k}^{(k)}} \cdots z_{N, k}^{\mathbf{X}_{N}^{(k)}}\right) \mid \mathbf{X}^{(0)}=x_{1}, \ldots, \mathbf{X}_{N}^{(0)}=x_{N}\right\}= \\
& z_{1,0}^{x_{1}} \cdots z_{N, 0}^{x_{N}} \int_{t_{1}=0}^{\infty} e^{-\omega_{1} t_{1}} \int_{t_{2}=0}^{\infty} e^{-\omega_{2} t_{2}} \cdots \int_{t_{k-1}=0}^{\infty} e^{-\omega_{k-1} t_{k-1}} \\
& \sum_{n_{1}^{(1)}=0}^{\infty} e^{-\lambda t_{1}} \frac{\left(\lambda t_{1}\right)^{n_{1}^{(1)}}}{n_{1}^{(1) !}} z_{1,1}^{n_{1}^{(1)}}\left[\prod_{j=1}^{N} \sum_{n_{j+1}^{(1)}=0}^{x_{j}}\left[\begin{array}{c}
x_{j} \\
n_{j}^{(1)}+1
\end{array}\right] p(j)^{n_{j+1}^{(1)}}(1-p(j))^{x_{j}-n_{j+1}^{(1)}} z_{j+1,1}^{n_{j+1}^{(1)}}\right]
\end{aligned}
$$

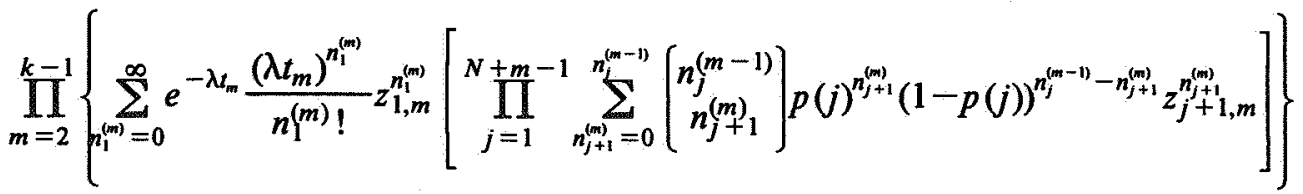

$$
\begin{aligned}
& {\left[1+\beta\left\{\omega_{k}+\lambda\left(1-z_{1, k}\right)\right\}\right]^{-\left(1+n_{1}^{(k-1)}+\ldots+n_{N+k-1}^{(k-1)}\right)} \prod_{j=1}^{N+k-1}\left(p(j) z_{j+1, k}+1-p(j)\right)^{n_{j}^{(k-1)}}} \\
& d B\left(t_{k-1}\right)^{\left(1+n_{1}^{(k-2)}+\ldots+n_{N+k-2}^{(k-2)}\right)^{*}} \cdots d B\left(t_{2}\right)^{\left(1+n_{1}^{(1)}+\ldots+n_{N+1}^{(1)}\right)^{*}} d B\left(t_{1}\right)^{\left(1+x_{1}+\ldots+x_{N}\right)^{*}}=
\end{aligned}
$$


15

$$
\begin{aligned}
& z_{1,0}^{x_{1}} \cdots z_{N, 0}^{x_{N}} A_{k}^{N}(1, \omega, z) \int_{t_{1}=0}^{\infty} e^{-\omega_{1} t_{1}} \int_{t_{2}=0}^{\infty} e^{-\omega_{2} t_{2}} \cdots \int_{t_{k-1}=0}^{\infty} e^{-\omega_{k-1} t_{k-1}} \\
& \sum_{n_{1}^{(1)}=0}^{\infty} e^{-\lambda t_{1}} \frac{\left(\lambda t_{1}\right)^{n_{1}^{(1)}}}{n_{1}^{(1) !}} z_{1,1}^{n_{1}^{(1)}}\left[\prod_{j=1}^{N} \sum_{n_{j+1}^{(1)}=0}^{x_{j}}\left[\begin{array}{c}
x_{j} \\
n_{j}^{(1)}
\end{array}\right] p(j)^{n_{j+1}^{(1)}}(1-p(j))^{x_{j}-n_{j+1}^{(1)}} z_{j+1,1}^{n_{j+1}^{(1)}}\right]
\end{aligned}
$$

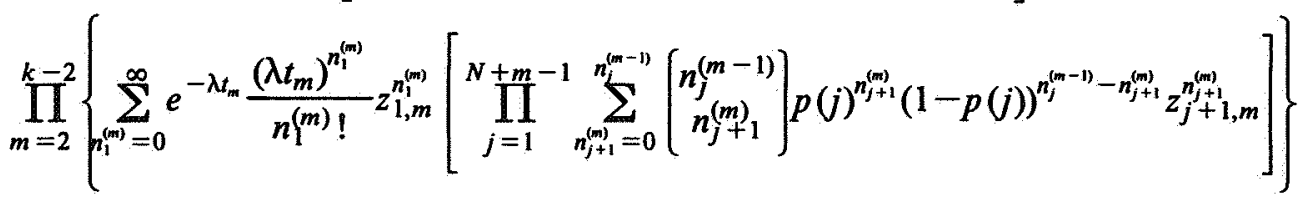

$$
\begin{aligned}
& e^{-\lambda t_{k-1}\left(1-z_{1, k-1} A_{k}^{N}(1, \omega, z)\left[p(1) z_{2, k}+1-p(1)\right]\right)} \\
& \prod_{j=1}^{N+k-2}\left(A_{k}^{N}(1, \omega, z)\left[p(j+1) z_{j+2, k}+1-p(j+1)\right] p(j) z_{j+1, k-1}+1-p(j)\right)^{n_{j}^{(k-2)}} \\
& d B\left(t_{k-1}\right)^{\left(1+n_{1}^{(k-1)}+\ldots+n_{k+k-2}^{(k-2)}\right)^{*}} \cdots d B\left(t_{2}\right)^{\left.\left(1+n_{1}^{(1)}+\ldots+n_{N+1}^{(1)}\right)\right)^{*}} d B\left(t_{1}\right)^{\left(1+x_{1}+\ldots+x_{k}\right)^{*}} .
\end{aligned}
$$

Next the integral with respect to $t_{k-1}$ is evaluated, yielding

$$
\begin{aligned}
& E\left\{e^{-\left(\omega_{1} \mathbf{S}_{1}+\ldots+\omega_{k} \mathbf{S}_{k}\right)}\left(z_{1,0}^{\mathbf{X}_{1}^{(0)}} \cdots z_{N, 0}^{\mathbf{X}_{N}^{(0)}}\right) \cdots\left(z_{1, k}^{\mathbf{X}_{1}^{(k)}} \cdots z_{N, k}^{\mathbf{X}_{N}^{(k)}}\right) \mid \mathbf{X}_{1}^{(0)}=x_{1}, \ldots, \mathbf{X}_{N}^{(0)}=x_{N}\right\}= \\
& z_{1,0}^{x_{1}} \cdots z_{N, 0}^{x_{N}} A_{k}^{N}(1, \omega, z) \int_{t_{1}=0}^{\infty} e^{-\omega_{1} t_{1}} \int_{t_{2}=0}^{\infty} e^{-\omega_{2} t_{2}} \cdots \int_{t_{k-2}=0}^{\infty} e^{-\omega_{k-2} t_{k-2}} \\
& \sum_{n_{1}^{(1)}=0}^{\infty} e^{-\lambda t_{1}} \frac{\left(\lambda t_{1}\right)^{n_{1}^{(1)}}}{n_{1}^{(1) !}} z_{1,1}^{n_{1}^{(1)}}\left[\prod_{j=1}^{N} \sum_{n_{j+1}^{n_{1}}=0}^{x_{j}}\left[\begin{array}{c}
x_{j} \\
n_{j}^{(1)}
\end{array}\right] p(j)^{\left.n_{j+1}^{(1)}(1-p(j))^{x_{j}-n_{j+1}^{(j)}} z_{j+1,1}^{n_{j}^{(1)}}\right]}\right] \\
& \prod_{m=2}^{k-2}\left\{\sum_{\sum_{i}^{(m)}=0}^{\infty} e^{-\lambda t_{m}} \frac{\left(\lambda t_{m}\right)^{n_{1}^{(m)}}}{n^{(m)} !} z_{1, m}^{n_{1}^{(m)}}\left[N \prod_{j=1}^{N+m-1} \sum_{n_{j+1}^{(m)}=0}^{\sum^{(m-1)}}\left[\begin{array}{c}
n_{j}^{(m-1)} \\
n_{j}^{(m)}
\end{array}\right] p(j)^{n_{j+1}^{(m)}}(1-p(j))^{h_{j}^{(m-1)}-n_{j+1}^{(m)}} z_{j+1, m}^{n_{j}^{(m)}}\right]\right\} \\
& {\left[1+\beta\left\{\omega_{k-1}+\lambda-\lambda z_{1, k-1} A_{k}^{N}(1, \omega, z)\left[p(1) z_{2, k}+1-p(1)\right]\right\}\right]^{-\left(1+n_{1}^{(k-2)}+\ldots+n_{N+k-2}^{(k-2)}\right)}} \\
& \prod_{j=1}^{N+k-2}\left(A_{k}^{N}(1, \omega, z)\left[p(j+1) z_{j+2, k}+1-p(j+1)\right] p(j) z_{j+1, k-1}+1-p(j)\right)^{n_{j}^{(k-2)}} \\
& d B\left(t_{k-2}\right)^{\left(1+n_{1}^{(k-3)}+\ldots+n_{N+k-3}^{(k-3)}\right)^{*}} \cdots d B\left(t_{2}\right)^{\left(1+n_{1}^{(1)}+\ldots+n_{N+1}^{(i)}\right)^{*}} d B\left(t_{1}\right)^{\left(1+x_{1}+\ldots+x_{k}\right)^{*}}= \\
& z_{1,0}^{x_{1}} \cdots z_{N, 0}^{x_{N}} A_{k}^{N}(1, \omega, z) A_{k}^{N}(2, \omega, z) \int_{t_{1}=0}^{\infty} e^{-\omega_{1} t_{1}} \int_{t_{2}=0}^{\infty} e^{-\omega_{2} t_{2}} \cdots \int_{t_{k-2}=0}^{\infty} e^{-\omega_{k-2} t_{k-2}} \\
& \sum_{n_{1}^{(1)}=0}^{\infty} e^{-\lambda t_{1}} \frac{\left(\lambda t_{1}\right)^{n_{1}^{(1)}}}{n_{1}^{(1)} !} z_{1,1}^{n_{1}^{(1)}}\left[\prod_{j=1}^{N} \sum_{n_{j+1}^{(j)}=0}^{x_{j}}\left[\begin{array}{c}
x_{j} \\
n_{j}^{(1)}+1
\end{array}\right] p(j)^{n_{j+1}^{(1)}}(1-p(j))^{x_{j}-n_{j+1}^{(1)} z_{j+1,1}^{n_{j}^{(1)}}}\right]
\end{aligned}
$$

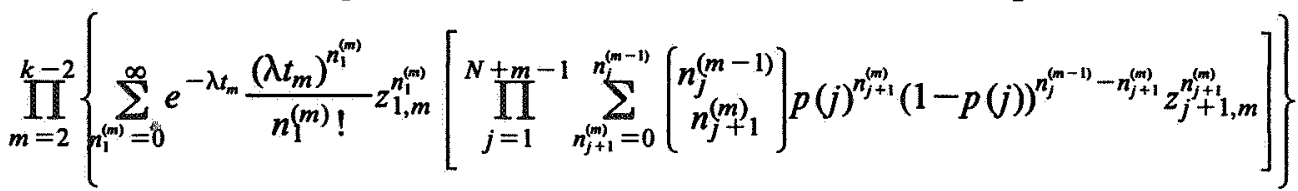


$\prod_{j=1}^{N+k-2}\left(A_{k}^{N}(2, \omega, z)\left[A_{k}^{N}(1, \omega, z)\left[p(j+1) z_{j+2, k}+1-p(j+1) \mid p(j) z_{j+1, k-1}+1-p(j)\right]\right)^{n_{j}^{(k-2)}}\right.$
$d B\left(t_{k-2}\right)^{\left(1+n_{1}^{(k-3)}+\ldots+n_{N+k-3}^{(k-3)}\right)^{*}} \cdots d B\left(t_{2}\right)^{\left(1+n_{1}^{(j)}+\ldots+n_{N+1}^{(j)}\right)^{*}} d B\left(t_{1}\right)^{\left(1+x_{1}+\ldots+x_{k}\right)^{*}}$.

We now sum over $n^{(k-2)}, \ldots, n_{N+k-2}^{(k-2)}$ and subsequently integrate with respect to $t_{k-2}$, thus obtaining $A_{k}^{N}(3, \omega, z)$ terms; etc.; finally the summations over $n_{l}^{(1)}, \ldots, n_{N}^{(1)}$ and the integration over $t_{1}$ are performed, which give rise to the $\left(f_{k}^{N}(i, \omega, z)\right)^{x_{i}}$ contribution in Formula (3.4). 